University of Nebraska - Lincoln

DigitalCommons@University of Nebraska - Lincoln

Papers in Veterinary and Biomedical Science

Veterinary and Biomedical Sciences,

Department of

September 2000

\title{
HSV Latency-Associated Transcript and Neuronal Apoptosis
}

Anthony B. Nesburn

Ophthalmology Research Laboratories, Cedars-Sinai Medical Center, Burns \& Allen Research Institute, Los Angeles, $C A$

Clinton J. Jones

University of Nebraska - Lincoln, cjones2@unl.edu

Follow this and additional works at: https://digitalcommons.unl.edu/vetscipapers

Part of the Veterinary Medicine Commons

Nesburn, Anthony B. and Jones, Clinton J., "HSV Latency-Associated Transcript and Neuronal Apoptosis" (2000). Papers in Veterinary and Biomedical Science. 86.

https://digitalcommons.unl.edu/vetscipapers/86

This Article is brought to you for free and open access by the Veterinary and Biomedical Sciences, Department of at DigitalCommons@University of Nebraska - Lincoln. It has been accepted for inclusion in Papers in Veterinary and Biomedical Science by an authorized administrator of DigitalCommons@University of Nebraska - Lincoln. 


\section{HSV Latency-Associated Transcript and Neuronal Apoptosis}

In a study of the latency-associated transcript ( $L A T)$ gene in herpes simplex virus-type 1 (HSV-1), Perng et al. reported that "in rabbit trigeminal ganglia [TGs], extensive apoptosis occurred with $L A T^{-}$virus but not with $L A T^{+}$ viruses," and concluded that the $L A T$ gene "promotes neuronal survival after HSV-1 infection by reducing apoptosis" (1). That conclusion rested on interpretations of two in situ analyses: (i) immunohistochemical staining for cleaved poly (ADP-ribose) polymerase (p85 PARP), and (ii) terminal deoxynucleotidyl transferasemediated deoxyuridine triphosphate nick-end labeling (TUNEL) to detect fragmented DNA. Cleavage of PARP by caspases between amino acids 214 and 215 generates an $89-\mathrm{kD}$ protein, in an early and diagnostic event in apoptosis (2). Perng et al. used a commercially available antibody (Anti-PARP p85 fragment pAb kit; Promega, Madison, Wisconsin) shown to be specific for the cleaved form of human and bovine PARP by Western blot analysis. Surprisingly, however, we found no published reports demonstrating that the same antibody is reactive against the cleaved form of PARP in rabbits, and our own Western blot and immunohistochemical analyses (Fig. 1) revealed that the antibody recognizes neither rabbit nor mouse p89 PARP protein.

In our experiments, protein extracts were produced from normal cells and from cells induced to undergo apoptosis [apoptosis was confirmed by characteristic DNA laddering (Fig. 1) and supported by positive TUNEL staining (Fig. 2)]. The extracts were electrophoresed, electroblotted, and probed with monoclonal antibody C-2-10 (Calbiochem, La Jolla, California), which detects both the intact $(116 \mathrm{kD})$ and cleaved $(89 \mathrm{kD})$ forms of PARP in bovine, human, mouse, rat, monkey, and rabbit cells (3), and with the Promega Anti-PARP p85 antibody. All protein extracts reacted with C-2-10 to yield bands of the expected sizes, and cleaved PARP was readily detected with C-210 in extracts from mouse, rabbit, and human cells undergoing apoptosis. By contrast, only the human cell extracts reacted with Promega Anti-PARP p85 (Fig. 1A). Likewise, when Anti-PARP p85 was employed for immunohistochemistry, only the apoptotic human cells showed the characteristic intense nuclear staining (Fig. 1B). As expected, the C-2-10 antibody stained both normal and apoptotic cells. We note that Perng et al. reported cytoplasmic as well as nuclear staining with Anti-PARP p85, whereas cleaved PARP is localized to the nucleus (4). We conclude that the signal detected by Perng et al. was unrelated to p85 PARP.

The sequence of the peptide employed to generate the Promega antibody is proprietary, but it is presumably similar to the 19 -amino acid peptide originally reported by Sallmann et al. (5). This peptide (amino acids 196 to 214 of human PARP) is identical in human, bovine, and chicken PARP but differs by three amino acids in the mouse protein. The sequence of rabbit PARP is not available, but the lack of immunoreactivity strongly suggests that the rabbit protein likewise contains differences in the amino acid sequence of this region sufficient to abrogate binding to Anti-PARP p85.

In a second in situ assay, Perng et al. used the Klenow-FragEL DNA fragmentation detection kit (Oncogene, Cambridge, Massachusetts) to detect fragmented DNA in apoptotic cells (1). The high positive correlation $(P<0.05)$ between the false positive signal obtained with the rabbit polyclonal Anti-PARP p85 on rabbit tissues and the TUNEL-positive cells (1) suggests that the TUNEL results may also have been misinterpreted. Factors such as calcium stores, active RNA transcription, and mRNA splicing can result in positive TUNEL signals that are unrelated to apoptosis (6). The subcellular localization of a positive signal resulting from fragmented DNA should be in the nucleus. However, the signal detected by Perng et al. in TG neurons appears to be from the cytoplasm, an unlikely localization for signal originating from fragmented DNA.

We examined TUNEL staining in human, mouse, and rabbit cells induced to undergo apoptosis (Fig. 2, A to C), as well as in sectioned rabbit TGs treated to serve as a positive control for TUNEL, as suggested by the kit manufacturer (Fig. 2, D to F). Positive TUNEL signal shows up as strong nuclear staining resembling the staining Perng et al. observed in infiltrating immune cells [figure
Fig. 1. Tests of immunoreactivity of Promega Anti-PARP p85 antibody with cleaved mouse, rabbit, and human PARP. (A) Western blot test. Swiss Webster mouse NIH/ 3T3 cells [American Type Culture Collection (ATCC)], primary young rabbit kidney (YRK) cells at passage 3 , and human Hela cells (ATCC) were induced to undergo apoptosis by treatment with staurosporine (Stauro, $0.2 \mu \mathrm{M}$ ) for 3 hours or sorbital (Sorb, 1 M) for 1 hour followed by 2 hours in normal medium. Both methods of inducing apoptosis yielded typical DNA fragmentation patterns with all cell types; right-hand panel shows example from YRK cells. Protein extracts were prepared, electrophoresed under reducing/denaturing conditions, electroblotted onto nitrocellulose membranes, and probed with the Calbiochem monoclonal antibody C-2-10 or with Promega Anti-PARP p85 essentially as described in (8). Extracts from cells that had undergone apoptosis (denoted by + symbol in row labeled Apoptotic) all showed bands of the expected sizes, indicating presence of both intact (116 $\mathrm{kD}$ ) and cleaved ( $89 \mathrm{kD}$ ) PARP, when tested with the C-2-10 antibody; by contrast, only the extract from the apoptotic human cell line tested positive for cleaved PARP using the Promega Anti-PARP p85 antibody. Similar findings (not shown) were obtained with Balb/C 3T3 (another mouse cell line), rabbit skin cells, and two additional human cell lines [HL60 and SK-N-SH neuroblastoma cells (ATCC)]. (B) Immunohistochemical-staining test. Untreated cells $(-)$ or cells induced to undergo apoptosis $(+)$ using staurosporine as described above were rinsed with phosphate-buffered saline solution, fixed with $4 \%$ paraformaldehyde, and processed for immunohistochemical detection of PARP using C-2-10 or AntiPARP p85, as suggested by the manufacturers. Positive signal is seen as a brown precipitate. As in Fig. $1 \mathrm{~A}$, all cells show a positive PARP signal when tested with the Calbiochem antibody, but only apoptotic human cells test positive with the Promega antibody. 
TECHNICAL COMMENT
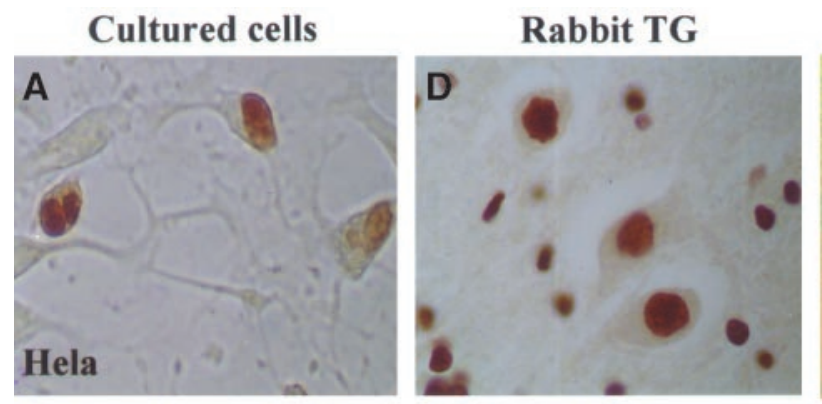

Perng et al. fig. 1
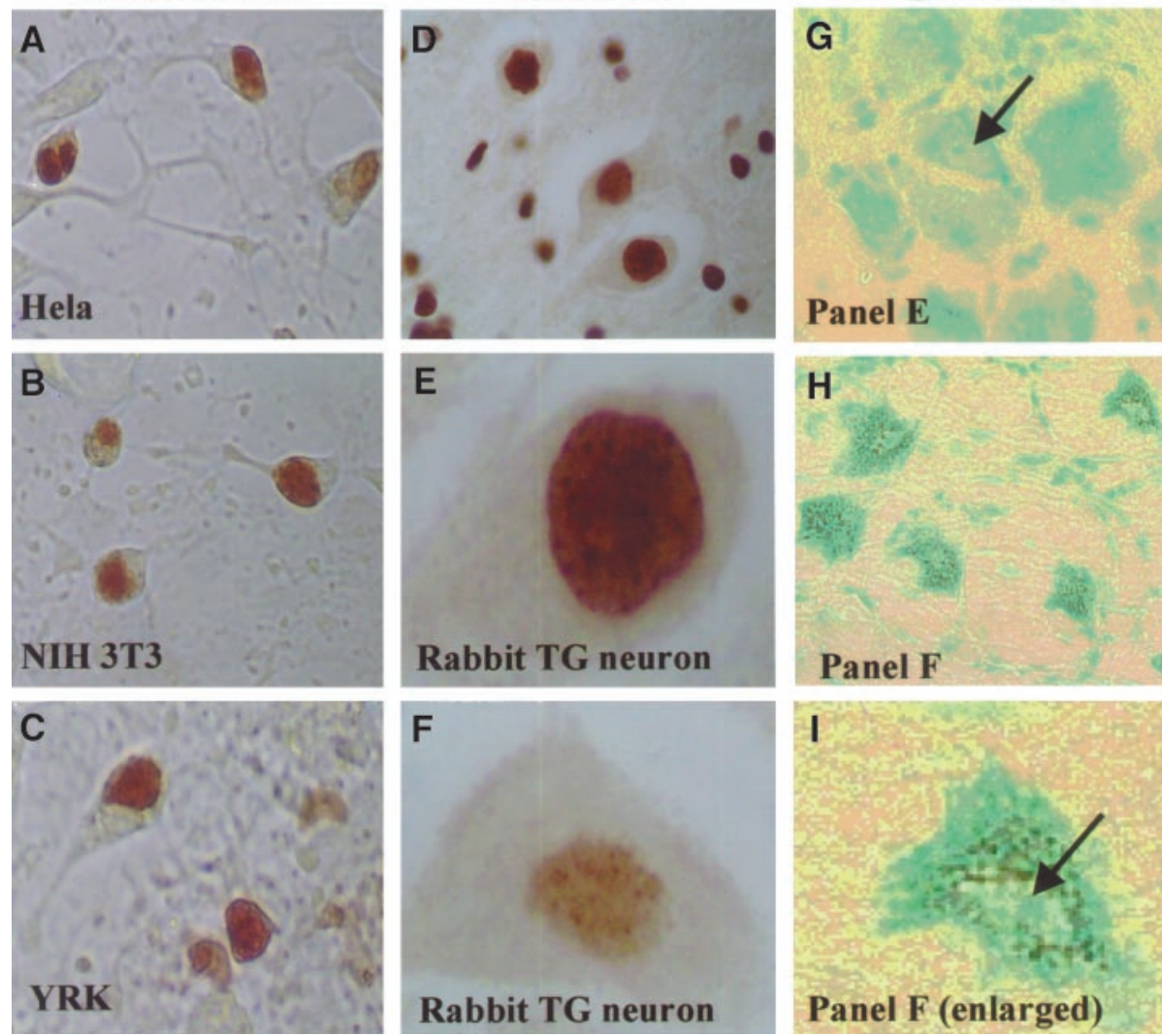
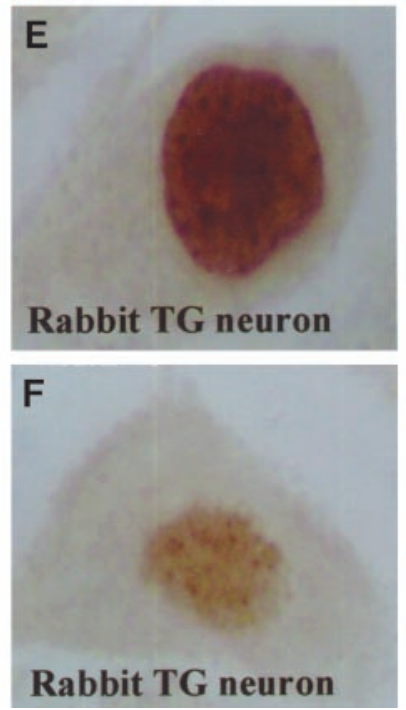
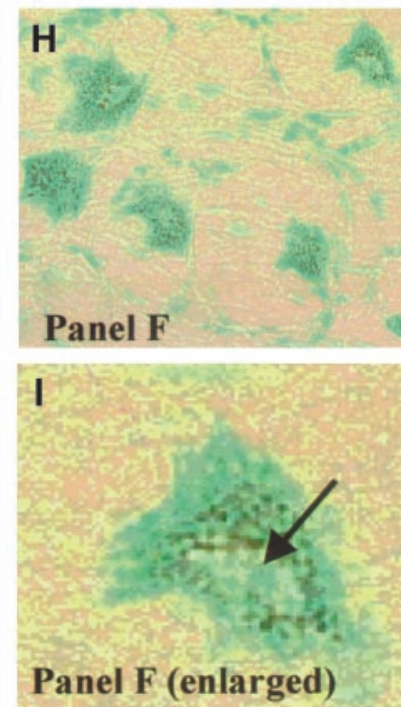

Fig. 2. TUNEL staining for fragmented DNA. Left panels: Additional cultures from (A) human, (B) mouse, and $(\mathbf{C})$ rabbit cell lines tested in Fig. 1 were processed for TUNEL according to instructions by the manufacturer (Promega). Cells were rarely positive in the absence of treatment, but the number of positive cells increased dramatically in cultures induced to undergo apoptosis. Positive signal is seen as a brown precipitate confined to the nucleus. Central panels: Rabbit TGs [(D) to (F)] were obtained at necropsy, drop-fixed in $4 \%$ paraformaldehyde, and processed as a positive control for TUNEL as described by the manufacturer (Promega). Magnifications are similar to those described for figure 1 of (1). Positive signal is seen as a brown precipitate confined to the nucleus. Right panels: Details from figure 1 of (1). (G) Figure 1E of (1) shows area interpreted as neuronal nucleus with prominent nucleolus (arrow) within larger blue-green area interpreted as cytoplasm. (H) Figure $1 \mathrm{~F}$ of (1), at original magnification. Brown dots are TUNEL-positive regions. (I) Figure $1 \mathrm{~F}$ of (1), at increased magnification. TUNEL-positive areas appear to be largely confined to area interpreted as cytoplasm, with little signal in area interpreted as nucleus (arrow).

$1 \mathrm{C}$ of (1)]. For comparison, two TUNEL results shown by Perng et al. [figure 1, E and $\mathrm{F}$ of $(1)]$ are reproduced here at several magnifications (Fig. 2, G to I). In figure 1E of (1), a neuronal nucleus with a prominent nucleolus is visible within a larger bluegreen region (arrow in Fig. 2G). Figure 1F of (1), which shows a positive TUNEL signal over the entire blue-green area of several neurons (Fig. 2H), apparently appears in (1) at a magnification approximately $30 \%$ smaller than that of two other panels from the same experiment (7). When the figure is enlarged to a similar magnification (Fig. 2I), the brown precipitate detected in the neurons looks primarily cytoplasmic, with what appears to be the nucleus (arrow in Fig. 2I) largely devoid of signal.

In summary, the antibody employed by Perng et al. (1) to identify apoptotic rabbit
TG neurons does not recognize the cleaved form of rabbit PARP. Although TUNELpositive cells were detected in rabbit TGs, most if not all of the nuclear signal shown appears to have been in infiltrating immune cells, as would be expected during the resolution of any infection. Thus, the report by Perng et al. (1) provides no evidence that the HSV-1 LAT gene blocks virus-induced apoptosis in neurons.

\section{R. L. Thompson}

Department of Molecular Genetics

University of Cincinnati Medical Center

Cincinnati, OH 45267-0524, USA

E-mail: Richard.Thompson@UC.edu

N. M. Sawtell

Division of Infectious Diseases Children's Hospital Medical Center

Cincinnati, OH 45229-3039, USA E-mail: Sawtn0@chmcc.org
References and Notes

1. G.-C. Perng et al., Science 287, 1500 (2000).

2. P. J. Duriez and G. M. Shah, Biochem. Cell Biol. 75, 337 (1997).

3. T. A. Holly et al., J. Mol. Cell Cardiol. 31, 1709 (1999).

4. M. Knaapen, M. De Bie, J. Muhring, M. Kockx, Promega Notes 72, 7 (1999).

5. F. R. Sallmann, S. Bourassa, J. Saint-Cyr, G. G. Poirier, Biochem. Cell Biol. 75, 451 (1997).

6. M. M. Kockx, J. Muhring, M. W. Knaapen, G. R. de Meyer, Am. J. Pathol. 152, 885 (1998).

7. Magnification of figure $1, D$ to $F$ of (1) was compared by measurement of the oblong blue-green areas that presumably are support cells or their nuclei and of the circular artifact present in the lower left quadrant of each of the three panels.

8. H. Towbin, T. Staehelin, J. Gordon, Proc. Natl. Acad. Sci. U.S.A. 76, 4350 (1979).

9. This work was supported by grant R01 Al32121 from the NIH.

\section{April 2000; accepted 5 July 2000}

Response: Thompson and Sawtell report that the Promega Anti-PARP p85 antibody did not recognize cleaved PARP in mouse or rabbit cells in their experiments, and conclude that the results reported with this antibody by Perng et al. (1) are an artifact. The Promega antibody was generated against a peptide based on the sequence of human $\mathrm{p} 85$. Although the corresponding bovine sequence differs by two amino acids, the antibody reacts with both human and bovine $\mathrm{p} 85$ (2). The mouse and rat sequences for this region of p85 differ from the human sequence by a single amino acid that corresponds to one of the bovine amino acid differences. External testers have successfully stained mouse and rat p85 using Promega Anti-PARP p85 (2). Thus, the negative mouse results reported by Thompson and Sawtell are surprising, and call into question the validity of their negative rabbit results.

Extracts that we prepared (Fig. 1) from rabbit skin cells induced to undergo apoptosis by staurosporin (lane RS-S) contained a band of approximately $85 \mathrm{kD}$ that was recognized by Anti-PARP p85, and that comigrated with the p85 band induced in human Jurkat cells by staurosporin (lane Jurkat-S) or anti-Fas antibody (lane Jurkat-F). Clearly, then, the Promega antibody recognizes the rabbit cleaved PARP p85 protein, and the argument to the contrary by Thompson and Sawtell has no merit. Their negative mouse and rabbit results apparently stemmed from technical problems, a bad batch of antibody, or some other unknown factor.

Thompson and Sawtell also contend that the Perng et al. results are incorrect because immunohistochemical tests of some neurons showed p85 staining in both the cytoplasm and the nucleus, whereas cleaved PARP should be found only in the nucleus. However, the proteolytic cleavage product of PARP has been detected immunocytochemically and confirmed by Western analysis both in the nuclei and in the cytoplasm of cells in a human osteosarcoma cell line that has under- 


\section{TECH N I A L COMMEN T}

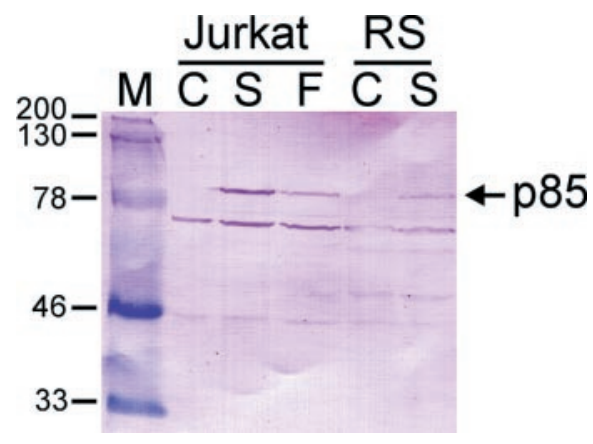
Jurkat cells induced for apoptosis with anti-Fas antibody; RS-C, control untreated rabbit skin cells; and RS-S, rabbit skin cells induced for apoptosis with staurosporin. Arrow indicates the PARP p85 band.

Fig. 2. Photomicrographs of TUNELstained TG sections. (A) Uninfected section. (B) Wild-type McKrae $\left(L A T^{+}\right)$. (C to G) dLAT2903 (LAT-). (A) to (C) correspond to figure $1, D$ to $F$, of (1); (D) to (G) are from additional experiments not shown in (1). Solid arrows indicate some of the TUNEL-positive neuronal nuclei; open arrow in (E) shows a TUNEL-negative neuron. $(G)$ is higher magnification image of boxed portion of $(F)$.

gone apoptosis in culture (3). Cytoplasmic staining for PARP has also been reported in groups of neurons in the human central nervous system (4). Thus, contrary to the assertion by Thompson and Sawtell, cleaved PARP can be found both in the cytoplasm and the nucleus during apoptosis.

In a similar vein, Thompson and Sawtell argue that DNA fragmentation indicated by TUNEL staining should be limited to the nucleus. A number of lines of evidence, however, strongly suggest that fragmented DNA and TUNEL staining can occur in the cytoplasm as well. In primary human keratinocytes and also in other cells undergoing apoptosis, cleaved DNA is extruded into the medium (5); the cleaved DNA obviously must pass through the cytoplasm to get out of the cell. In apoptotic cells in the developing retina of rabbits, rats, and cats, TUNEL staining labeled the cytoplasm (6), an indication that DNA fragments spread into the cytoplasm of the dying cell. In brains of Creutzfeldt-Jakob disease victims, nuclear DNA leakage produced positive TUNEL staining in the cytoplasm of some cells (7). TGs of newborn rats injected with capsaicin to induce apoptosis and DNA fragmentation showed a TUNEL signal that had spread from the neuronal nucleus to the cytoplasm $(8)$. The instructions in the commercially avail-
Fig. 1. Rabbit PARP p85 is recognized by Promega Anti-PARP p85 on Western blots. Rabbit skin cells (RS) and human Jurkat cells were induced to undergo apoptosis. Equal amounts of total cell extracts (30 $\mu \mathrm{g}$ of protein) were run on each lane of a $10 \%$ SDS-polyacrylamide gel, transferred to polyvinylidene difluoride membrane, incubated with Anti-PARP p85 antibody, washed, incubated with donkey anti-rabbit IgG conjugated to alkaline phosphatase, and developed with the reagent BCIP/NBT (Gibco BRL, Life Technologies, Rockville, Maryland). Lanes: $M$, molecular weight markers; Jurkat- $C$, control untreated Jurkat cells; Jurkat-S, Jurkat cells induced for apoptosis with staurosporin; Jurkat- $F$,

solid arrows). Both situations are consistent with the literature.

In summary, Thompson and Sawtell present no convincing evidence against the finding in (1) that $L A T$ is an anti-apoptosis gene. The results presented here clearly show that Anti-PARP p85 recognizes rabbit p85; the data in the comment on the Promega antibody's lack of reactivity with mouse p 85 disagrees with the statements of the kit manufacturer; the assumptions in the comment regarding the amino acid sequence against which the p 85 antibody was made are incorrect; and both PARP and TUNEL staining have been previously reported in both the nuclei and the cytoplasm of apoptotic cells. We note, too, that Thompson and Sawtell did not attempt to refute a separate key finding of Perng et al:: that a plasmid expressing LAT nucleotides 301 to 2659 efficiently blocks apoptosis in transient transfection assays (1). (This is particularly interesting, because the plasmid was originally kindly provided to us by R. L. Thompson, one of the comment authors.) These results unequivocally demonstrated that $L A T$ has a powerful anti-apoptosis activity - and that alone would suggest that $L A T$ can block apoptosis in rabbit trigeminal ganglia.

able kit Cell Death Detection ELISA (Cat. No. 1544 675, Roche, Mannheim, Germany), which detects apoptosis based on the presence of cytoplasmic histone-associated DNA fragments, state that "enrichment of mono- and oligonucleosomes in the cytoplasm of the apoptotic cell is due to the fact that DNA degradation occurs several hours before plasma membrane breakdown." Finally, HSV-1, the subject of the Perng et al. (1) study, buds through the nuclear membrane, a situation that may make the nuclear membrane more susceptible to leakage of fragmented DNA.

In the TUNEL controls of Thompson and Sawtell (their figure 2, D to F), none of the digested DNA moved out of the nucleus, because these cells were fixed before deoxyribonuclease (DNase) treatment. In contrast, when the DNA is fragmented in live cells undergoing apoptosis, small DNA fragments can exit the nucleus and be stained in the cytoplasm. (The DNase control is intended to show that the kit reagents are functioning properly; it is not meant as a positive control for all biological situations.) Moreover, although in some neurons shown in (1) TUNEL signal appeared in both the nucleus and the cytoplasm, in other samples not shown in (1) TUNEL staining was unequivocally detected only in the nucleus (Fig. 2,
Steven L. Wechsler

Guey-Chuen Perng

Homayon Ghiasi

Anthony B. Nesburn

Ophthalmology Research Laboratories Cedars-Sinai Medical Center Burns \& Allen Research Institute 8700 Beverly Boulevard

Los Angeles, CA 90048, USA and Department of Ophthalmology UCLA School of Medicine

Los Angeles, CA 90024, USA

Clinton Jones

Department of Veterinary and Biomedical Sciences

Center for Biotechnology

University of Nebraska

Lincoln, NE 68583, USA

\section{References}

1. G.-C. Perng et al., Science 287, 1500 (2000).

2. T. Riss, Promega, personal communication.

3. D. S. Rosenthal et al., Exp. Cell Res. 232, 313 (1997).

4. M. R. Cookson, P. G. Ince, P. A. Usher, P. J. Shaw, Brain Res. 834, 182 (1999).

5. W. H. Tolleson et al., Carcinogenesis 17, 239 (1996).

6. R. Egensperger, J. Maslim, S. Bisti, H. Hollander, J. Stone, Dev. Brain Res. 97, 1 (1996).

7. I. Ferrer, Acta Neuropathol. (Berl.) 97, 5 (1999).

8. T. Sugimoto, A. Takeyama, C. Xiao, T. TakanoYamamoto, H. Ichikawa, Brain Res. 818, 147 (1999).

23 May 2000; accepted 27 July 2000 